\title{
Artro-ressonância do Ombro na Instabilidade Anterior
}

\section{Shoulder Magnetic Ressonance Arthrography to Evaluate Anterior Instability}

\author{
Eduardo Honda ${ }^{(1)}$, André Yui Aihara ${ }^{(1)}$, Jamil Natour ${ }^{(2)}$, Artur da Rocha Corrêa Fernandes ${ }^{(1)}$
}

\section{INTRODUÇÃO}

O ombro é a articulação com a maior capacidade e amplitude de movimento, tendo mais de 180 graus de amplitude em todos os planos. Devido a esta grande mobilidade, a articulação glenoumeral é uma das mais instáveis e freqüentemente luxadas do corpo, correspondendo a cerca de $50 \%$ de todas as luxações ${ }^{(1,2)}$.

Os estabilizadores estáticos do ombro são: glenóide óssea, lábio glenoidal, cápsula articular, ligamentos glenoumerais e intervalo dos rotadores (espaço entre a margem anterior do músculo supraespinhal e a margem superior do músculo subescapular, contendo no seu interior o tendão da cabeça longa do bíceps, ligamento coracoumeral e o ligamento glenoumeral superior).

Os estabilizadores dinâmicos são: músculos do manguito rotador e escapuloumerais. O balanço entre eles é responsável pela estabilidade da articulação do ombro ${ }^{(3,4,5)}$.

Para estudo dessas estruturas, a artrografia é o melhor método. A distensão da cápsula articular permite avaliar melhor sua morfologia, bem como do lábio glenoidal, dos ligamentos glenoumerais, do intervalo dos rotadores, da face articular do manguito rotador, de acompanhamento pós-operatório da articulação glenoumeral e do manguito rotador $^{(6)}$.

A artro-ressonância magnética constitui-se no método de imagem de escolha, pois permite a análise mais precisa das estruturas mencionadas, assim como dos demais componentes da articulação e também das estruturas relacionadas a essa articulação, como os ventres musculares dos componentes do manguito rotador e do tendão da cabeça longa do bíceps.

\section{INSTABILIDADE GLENOUMERAL}

A instabilidade do ombro é definida como o escorregamento da cabeça umeral para fora da cavidade glenoidal durante atividades, causando sintomas, podendo variar desde subluxação até luxação. Esta ocorre em cerca de $2 \%$ da população, sendo a maioria homens (85-90\%), com menos de 20 anos (88-95\%), durante eventos esportivos (75\%). Aproximadamente $25 \%$ dos pacientes relatam história familiar e alguns estudos demonstraram que a taxa de recorrência da lesão é alta, sendo maior quanto mais novo o paciente, acometendo praticamente $100 \%$ daqueles com até 10 anos de idade.

A classificação das instabilidades é baseada tradicionalmente na direção (anterior, posterior, inferior e multidirecional), no grau (subluxação, luxação e microtrauma), na freqüência (aguda, recorrente e crônica) e na etiologia (traumática, atraumática, microtrauma, congênita e neuromuscular).Na maioria das vezes, as instabilidades anteriores são diferenciadas em duas formas: TUBS (traumatic, unidirecional, Bankart lesion, surgery) e AMBRI (atraumatic, multidirecional, bilateral, rehabilitation, inferior capsular shift). O mecanismo de lesão habitual ocorre devido à queda sobre o braço em extensão, rotação externa e abdução ${ }^{(7)}$. Esses pacientes (pós-trauma) geralmente têm lesão da porção ântero-inferior do lábio glenoidal (Bankart ou suas variantes), e podem ter lesão de Hill-Sachs (Fratura por impactação da borda póstero-superior da cabeça umeral) associada (74\%). Outras lesões associadas descritas são: ruptura do manguito (13\%), corpos livres (14\%), fratura com avulsão glenoidal (4\%) e lesão do lábio glenoidal posterior (10\%). Uma possível complicação da luxação glenoumeral ântero-inferior é a lesão do nervo axilar ou de seus ramos, causando atrofia dos músculos por eles inervados ${ }^{(7,8)}$.

\footnotetext{
1. Departamento de Diagnóstico por Imagem da Universidade Federal de São Paulo (UNIFESP).

2. Disciplina de Reumatologia da UNIFESP

Endereço para Correspondência: Artur da Rocha Corrêa Fernandes, Departamento de Diagnóstico por Imagem da UNIFESP, Rua Botucatu, 740, CEP 04023-900, São Paulo, SP, Brasil.
} 


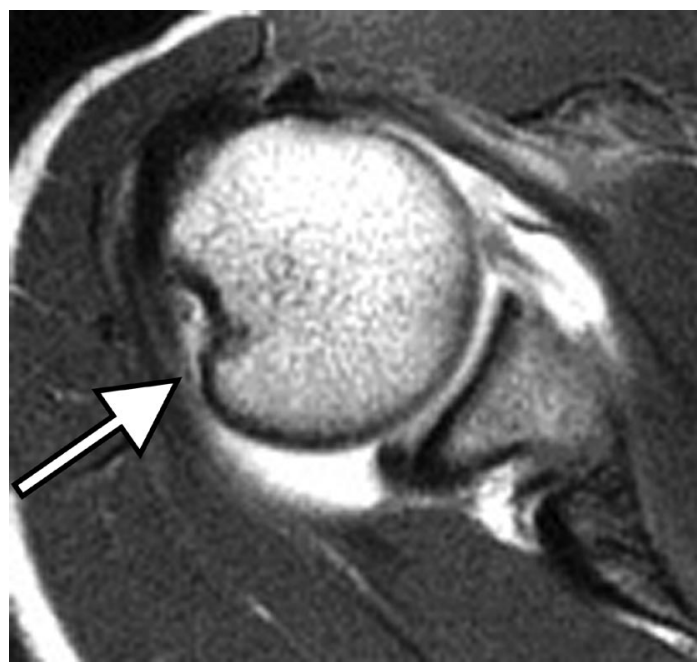

Figura 1 - Lesão de Hill-Sachs (seta).

\section{DIAGNÓSTICO POR IMAGEM}

As luxações podem ser avaliadas pela radiografia convencional, através dos achados de lesão de Hill-Sachs e Bankart ósseo. Na vigência de instabilidade anterior do ombro, é essencial que sejam obtidas, inicialmente, imagens radiográficas simples em posição neutra, em rotação interna e em rotação externa para se descartar a possibilidade de depósito de hidroxiapatita de cálcio no manguito rotador, que pode ser equivocadamente interpretado como extravasamento do meio de contraste na artro-ressonância e, assim, diagnosticar-se erroneamente ruptura tendínea ${ }^{(9)}$.

Ainda na fase aguda, devido à presença de derrame articular que promove distensão capsular e contraste natural, a ressonância magnética $(\mathrm{RM})$ convencional pode demonstrar precisamente a lesão labral resultante do deslocamento glenoumeral.

A artrotomografia é um método alternativo, nos casos em que o paciente está impedido de realizar RM ou artroressonância (claustrofóbicos, portadores de marca-passo e/ou próteses metálicas).

Dentre os métodos de imagem, aquele que oferece mais informações é a artro-ressonância, que é o método de escolha e, além disso, no caso de instabilidade crônica, é capaz de mostrar de maneira precisa a lesão do lábio glenoidal. Pode-se visibilizar também lesões associadas como a lesão de Hill-Sachs, lesões de manguito e condrais, além de corpos livres intra-articulares. Entretanto, é um método minimamente invasivo, em que é preciso injeção do meio de contraste intra-articular ${ }^{(10)}$.

A importância da artro-ressonância constitui-se em contribuir para a definição da conduta cirúrgica (plicatura, fixação do lábio glenoidal) ou também em estabelecer parâmetros de medidas no tratamento clínico, informando, por exemplo, a espessura dos ventres musculares.

\section{TÉCNICA DE ARTRO-RESSONÂNCIA}

Várias técnicas de injeção intra-articular de material de contraste foram descritas desde o seu início, em 1933. Tradicionalmente, a realização desta é feita através da fluoroscopia por acesso anterior, como fora descrito por Schneider et $a^{(8)}$. Esta técnica utiliza agulha 22 gauge que é introduzida verticalmente na junção dos terços médio/inferior da articulação glenoumeral. Pode-se, ainda, realizar a punção pela abordagem posterior ${ }^{(11)}$ ou através do intervalo dos rotadores ${ }^{(12,13)}$. A introdução de contraste pode ser feita através de fluoroscopia, ultra-sonografia (US) ${ }^{(14)}$ ou tomografia computadorizada (TC) ${ }^{(15)}$ e o exame de RM deve ser iniciado até 30 minutos após a injeção, evitando a perda da distensão capsular e absorção do meio de contraste. ${ }^{(9,16,17)}$
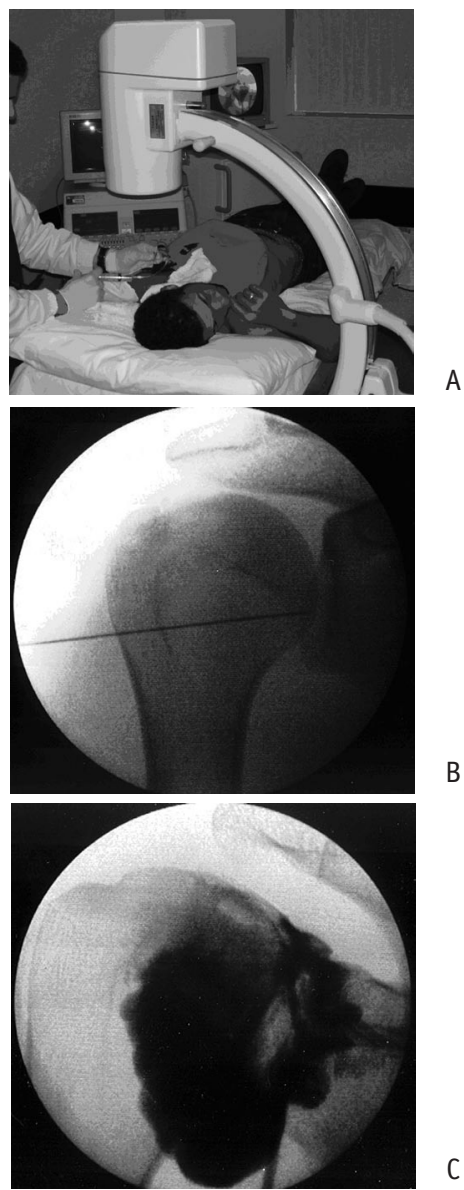

Figura 2 - Técnica de injeção intra-articular do meio de contraste pela fluoroscopia. A: técnica de punção anterior guiada pela fluoroscopia. B e C: imagem fluoroscópica demonstrando contraste intra-articular. 
As seqüências spin-echo ponderadas em $\mathrm{Tl}$ sem e com saturação de gordura, maximizam a intensidade do contraste. Seqüências spin-echo ponderadas em T2 com saturação de gordura ajudam na identificação de coleções líquidas extra-articulares, assim como cistos labrais e caracterização de lesões da medula óssea incidentais ou massas periarticulares. Seqüências spin-echo ponderadas em T2 com saturação de gordura podem demonstrar edema medular. ${ }^{(10,18-20)}$

Após a obtenção de imagens com o braço em posição neutra, complementa-se o exame com imagens adicionais com o braço em abdução e rotação externa (ABER), tensionando desta forma a banda anterior do ligamento glenoumeral inferior, aumentado a sensibilidade (de $48 \%$ para 96\%) e a especificidade (de 91 para 97\%) na identificação de lesões do complexo cápsulo-labral ântero-inferior ${ }^{(19-22)}$. A partir da imagem localizadora no plano coronal, as imagens em ABER são realizadas no plano paralelo ao eixo longitudinal do úmero ${ }^{(23)}$.

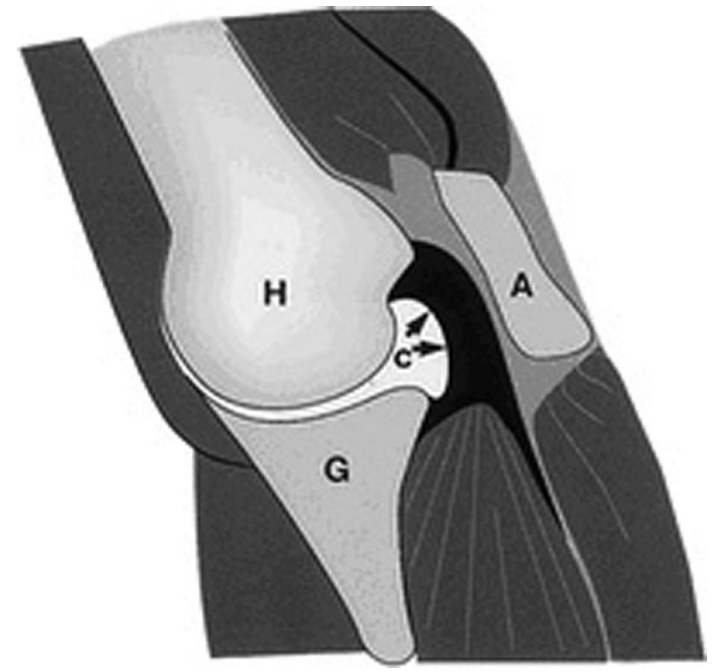

Figura 3 - Ilustração de ombro normal na posição ABER demonstrando a relação entre o úmero $(H)$, superfície glenoidal $(G)$, acrômio $(A)$, tendão do manguito rotador (setas) e cavidade articular (C). (Retirado de Horizontal component of partial-thickness tears of rotator cuff: imaging characteristics and comparison of ABER view with oblique coronal view at MR arthrography - initial results. Radiology: 224:470-6, 2002).
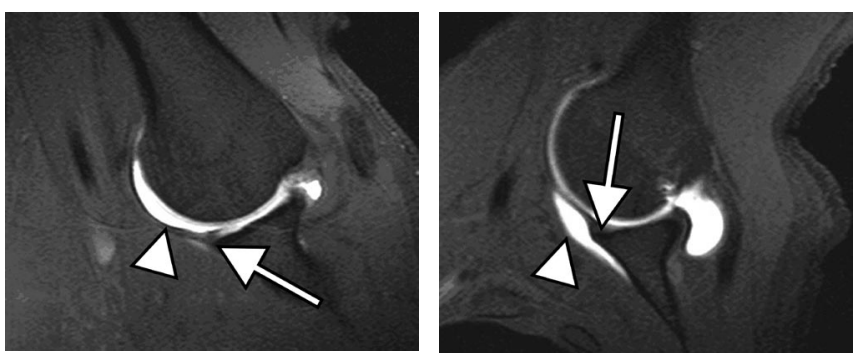

Figura 4 - Imagens em ABER mostrando o labrum ântero-inferior (seta) e a banda anterior do LGUI (ponta de seta), inserindo no labrum.

\section{ARTRO-RESSONÂNCIA E A INSTABILIDADE GLENOUMERAL ANTERIOR TRAUMÁTICA}

A forma mais comum de instabilidade ocorre após evento traumático, que geralmente é anterior, lesionando o labrum ântero-inferior, com ou sem lesão da glenóide óssea adjacente (lesão de Bankart e suas variantes).

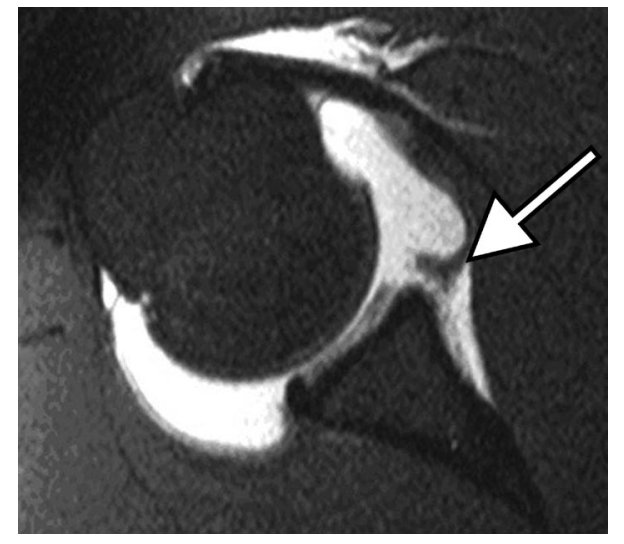

A

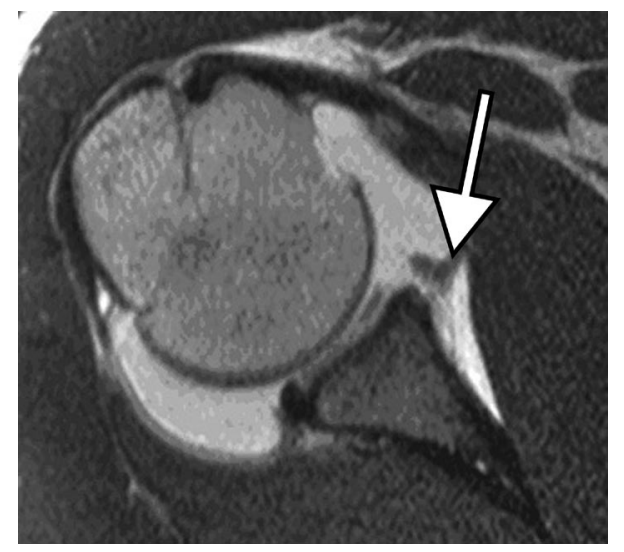

B

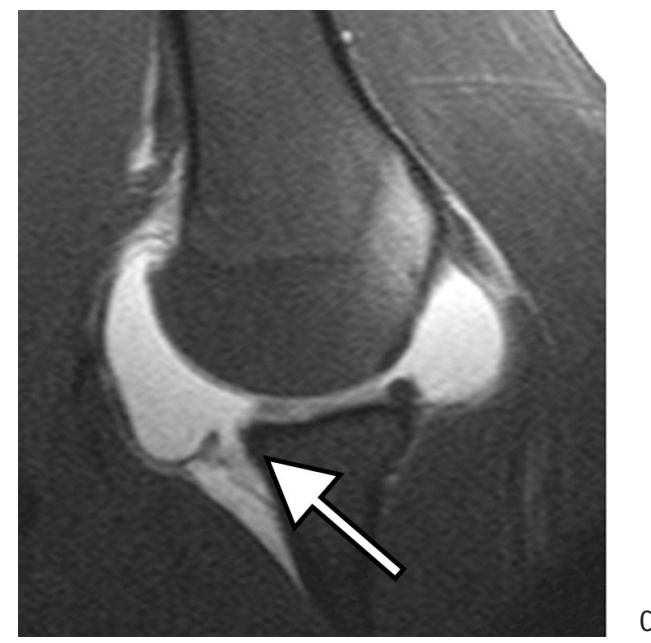

Figura 5 - Avulsão do labrum ântero-inferior e do periósteo, em relação à glenóide óssea (Bankart clássico). A: axial com SG. B: axial sem SG. C: ABER. 

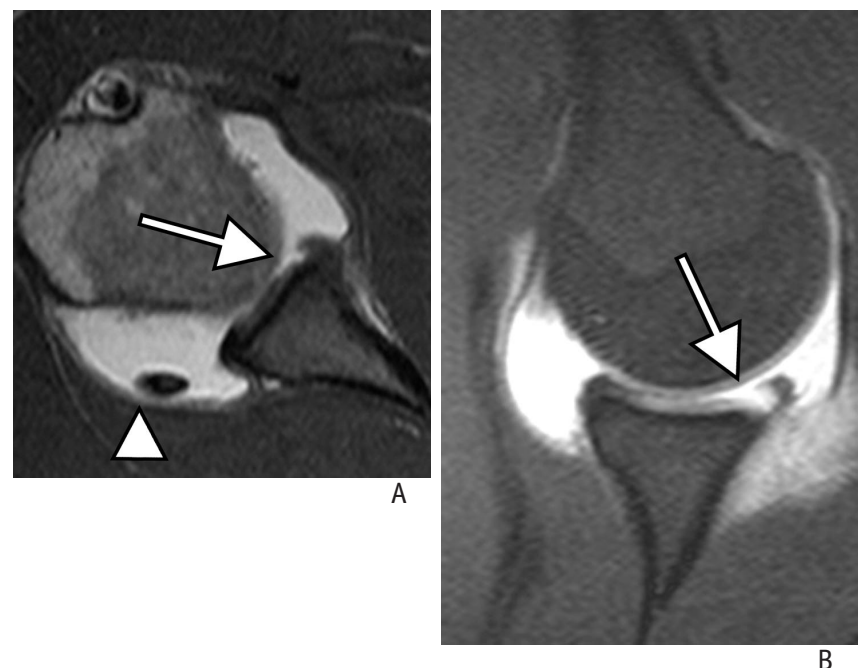

Figura 6 - Lesão Perthes. Periósteo escapular está íntegro, associado à avulsão do lábio anterior da glenóide. As imagens axial $(A)$ e em ABER (B) demonstram o deslocamento do labrum ântero-inferior com periósteo íntegro. Note o corpo livre intra-articular em A (seta).
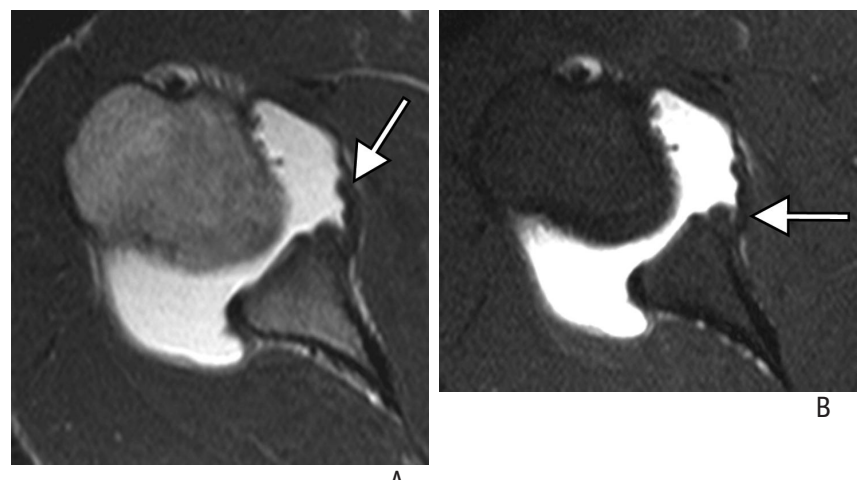

Figura 7 - Lesão ALPSA. Lesão por avulsão da bainha periosteal lábioligamentar anterior. Imagens axiais (A) sem e (B) com supressão de gordura, mostrando labrum destacado e aderido medialmente no colo da glenóide (seta). Note, também, o aspecto ondulado do LGUI.

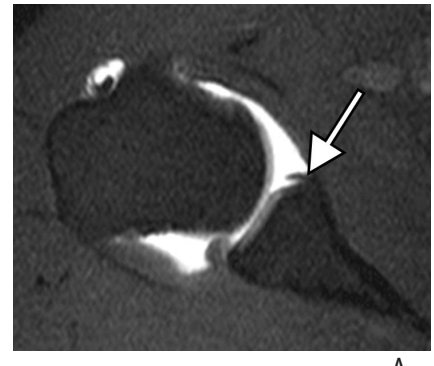

A

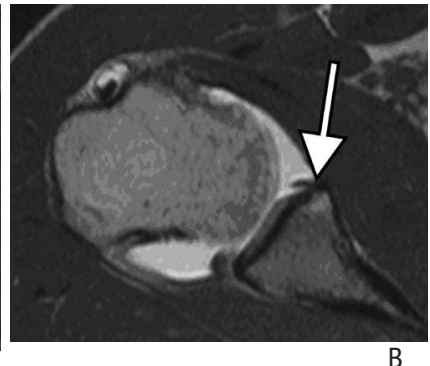

B
Figura 8 - Lesão GLAD. Lesão labial ântero-inferior superficial associada a uma lesão da cartilagem articular da glenóide ântero-inferior. Imagem demonstrando lesão labral associada à lesão da cartilagem glenoidal adjacente (seta).
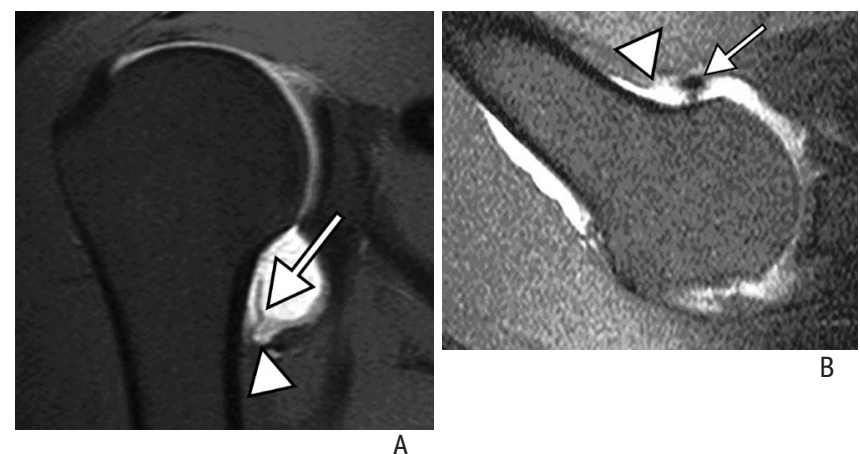

Figura 9 - Lesão HAGL. Avulsão da inserção umeral da banda anterior do LGUI, que pode estar associado à lesão do lábio glenoidal anterior. Imagem coronal (A) e em ABER (B) mostrando a lesão do LGUI na sua inserção umeral (seta) com contraste extravasando pelo defeito (ponta de seta).

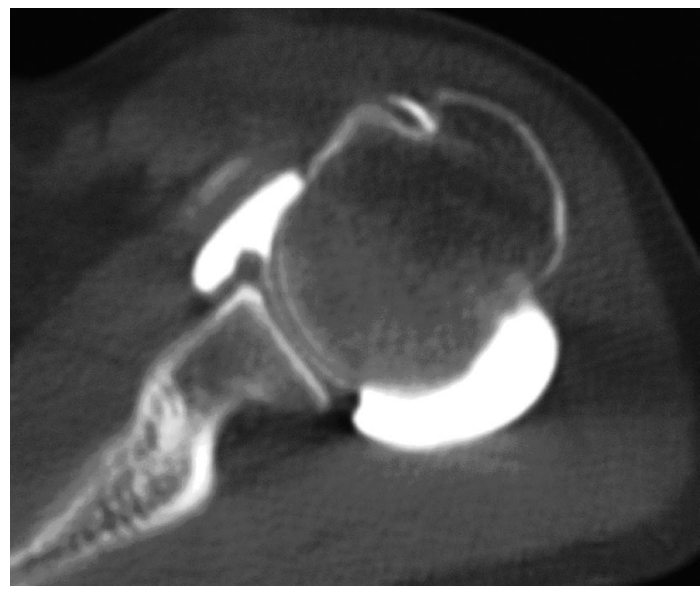

Figura 10 - Artrotomografia normal.
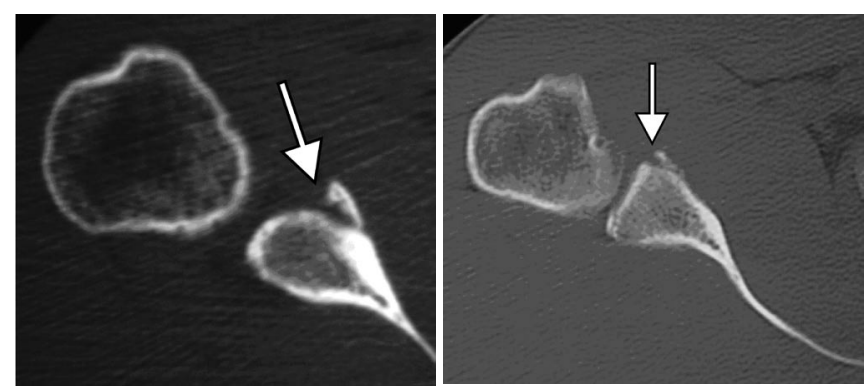

Figura 11 - Imagens TC axiais demonstrando lesão Bankart óssea (seta).

\section{CONCLUSÃO}

$\mathrm{Na}$ abordagem de paciente com queixa de instabilidade traumática ou atraumática, a avaliação por imagem deve ser iniciada por exame radiológico convencional, seguido por artro-ressonância, tendo como métodos alternativos a RM convencional e a artro-tomografia. 


\section{REFERÊNCIAS}

1. Beltran J, Kim DHM: MR imaging of shoulder instability injuries in the athlete. Magn Reson Imaging Clin N Am 11: 221-38, 2003

2. Parmar H, Jhankaria B, Maheshwari M et al: Magnetic resonance arthrography in recurrent anterior shoulder instability as compared to arthroscopy: A prospective comparative study. J Postgrad Med 48: 270-4, 2002.

3. Farber JM, Buckwalter KA: Sports- related injuries of the shoulder: instability. Magn Reson Imaging Clin N Am 10: 235-49, 2002.

4. Cohen M, Abdalla RJ (ed): Lesões nos Esportes Diagnóstico Prevenção e Tratamento. Revinter, 2003 - RJ.

5. Stoller DW: Ressonância Magnética em Ortopedia \& Medicina Desportiva. 2a. ed. Rio de Janeiro, Koogan, 2000.

6. Chandnani VP, Gagliardi JA, Murnane TG et al: Glenohumeral ligaments and shoulder capsular mechanism: Evaluation with MR arthrography. Radiology 196: 27-32, 1995.

7. Tirman PFJ: Shoulder Update. Shoulder Instability In Advanced MRI from head to toe - The Cutting Edge. Graz, Austria. May $1-3,2002$.

8. Schneider R, Ghehnar B, Kaye JJ: A simplified injection technique for shoulder arthrography. Radiology 114: 738-9, 1975.

9. Jacobson JA, Lin J, Jamadar DA, Hayes CW: Aids to successful shoulder arthrography performed with a fluoroscopically guided anterior approach. Radiographics 23: 373-9, 2003.

10. Chung CB, Corrente L, Resnick D: MR arthrography of the shoulder. Magn Reson Imaging Clin N Am 12: 25-38, 2004.

11. Farmer KD, Hughes PM: MR Arthrography of the shoulder: fluoroscopically guided technique using a posterior approach. AJR 178: 433-4, 2002.

12. Kwak SM, Brown RR, Trudell D, Resnick D: Glenohumeral joint: comparison of shoulder positions at MR arthrography. Radiology 208: 375-80, 1998.
13. Palmer WE, Caslowitz PL: Anterior shoulder instability: Diagnostic criteria determined from prospective analysis of 121 MR arthrograms. Radiology 197: 819-25, 1995.

14. Valls R, Melloni P: Sonographic guidance of needle position for MR arthrography of the shoulder. AJR 169: 845-7, 1997.

15. Habibian A, Stauffer A, Resnick D et al: Comparison of conventional and computed arthrotomography with MR imaging in the evaluation of the shoulder. J Comput Assist Tomogr 13: 968-75, 1989.

16. Chung CB, Dwek JR, Feng S, Resnick D: MR Arthrography of the glenohumeral joint: A tailored approach. AJR 177: 217-9, 2001.

17. Dépelteau H, Bureau NJ, Cardinal E, Aubin B, Brassard P: Arthrography of the shoulder: A simple fluoroscopically guided approach for targeting the rotator cuff interval. AJR 182: 329$32,2004$.

18. Steinbach LS, Palmer WE, Schweitzer ME: MR arthrography. Special focus session. Radiographics 22: 1223-46, 2002.

19. McCauley TR: MR imaging of the glenoid labrum. Magn Reson Imaging Clin N Am 12: 97-109, 2004.

20. Shankman S, Bencardino J, Beltran J: Glenohumeral instability: evaluation using MR arthrography of shoulder. Skeletal Radiol 28: 365-8,1999.

21. Bui-Mansfield LT, Taylor DC, Uhorchak JM, Tenuta JJ: Humeral avulsions of the glenohumeral ligaments: Imaging Features and a Review of the literature. AJR 179: 649-55, 2002.

22. Wischer TK, Bredella MA, Genant HK, Stoller DW, Bost FW, Tirman PFJ: Perthes lesion (a variant of the Bankart lesion): MR imaging and MR arthrographic findings with surgical correlation. AJR 178: 233-7, 2002.

23. Beltran J, Rosenberg ZS, Chandnani VP, Cuomo F, Beltran S, Rokito A: Glenohumeral instability: Evaluation with MR arthrography. Radiographics 17: 657-73, 1997. 